\section{Modelling malaria reduction in a highly endemic country: Evidence from household survey, climate, and program data in Zambia}

\author{
Mukumbuta Nawa, ${ }^{1}$ \\ Hikabasa Halwindi, ${ }^{2}$ Peter Hangoma ${ }^{1}$ \\ ${ }^{1}$ Department of Health Policy and \\ Management, and ${ }^{2}$ Department of \\ Community and Family Medicine, \\ University of Zambia, School of Public \\ Health, Lusaka, Zambia
}

\begin{abstract}
Substantial efforts have seen the reduction in malaria prevalence from $33 \%$ in 2006 to $19.4 \%$ in 2015 in Zambia. Many studies have used effect measures, such as odds ratios, of malaria interventions without combining this information with coverage levels of the interventions to assess how malaria prevalence would change if these interventions were scaled up. We contribute to filling this gap by combining intervention coverage information with marginal predictions to model the extent to which key interventions can bring down malaria in Zambia. We used logistic regression models and derived marginal effects using repeated cross-sectional survey data from the Malaria Indicator Survey (MIS) datasets for Zambia collected in 2010, 2012 and 2015. Average monthly temperature and rainfall data were obtained from climate explorer a satellite-generated database. We then conducted a counterfactual analysis using the estimated marginal effects and various hypothetical levels of intervention coverage to assess how different levels of coverage would affect malaria prevalence. Increasing IRS and ITNs from the 2015 levels of coverage of $28.9 \%$ and $58.9 \%$ respectively to at least $80 \%$ and rising standard housing to $20 \%$ from the $13.4 \%$ in 2015 may bring malaria prevalence down to below $15 \%$. If the percentage of modern houses were increased further to $90 \%$, malaria prevalence might decrease to $10 \%$. Other than ITN and IRS, streamlining and increasing of the percentage of standard houses in malaria fight would augment and bring malaria down to the levels needed for focal malaria elimination. The effects of ITNs, IRS and Standard housing were pronounced in high than low epidemiological areas.
\end{abstract}

\section{Introduction}

Malaria is endemic to Zambia, with highest prevalence during the rainy season from December to April. ${ }^{1}$ It is among the top causes of disability-adjusted life years (DALYs) lost and it is associated with substantial productivity losses in Zambia. ${ }^{2}$ In 2015 , the national malaria prevalence was $19.4 \%$ in children aged below five years with other parts of the country such as Luapula province in the north, having a prevalence as high as $32 \% .^{3}$ High malaria prevalence is associated with increased morbidity and mortality among children, chronic anaemia, stunting, and wasting. ${ }^{4,5}$

Substantial efforts have been put in place to bring down malaria, especially after the year 2000, when critical malaria and other interventions were rapidly scaled up following the millennium summit that agreed on the 2015 agenda for global development. This was aided by increases in development assistance for health and improvements in Zambia's economic performance, with economic growth averaging $7 \%$ per annum. ${ }^{6}$ The malaria program is coordinated by the National Malaria Elimination Program (NMEP) and guided by strategic plans (National Malaria Strategic Plans 2001 - 2005, 2006 - 2010, and 2011 - 2016).1,7 During this time, malaria prevalence reduced by at least $41.2 \%$ of the baseline, from $33 \%$ in 2006 to $19.4 \%$ in $2015 .{ }^{3}$ However, progress towards elimination is slow as the national prevalence of $19.4 \%$ in children is still within the high endemic category defined as malaria prevalence above $15 \%$ in under-five children. ${ }^{8}$ Importantly, there was an upsurge in malaria prevalence between 2010 and 2015 from $16 \%$ to $19.4 \% .^{9}$ This situation is concerning given that the elimination of the disease is now the government's goal in the short to medium term. ${ }^{7}$

There is a lot of literature examining the effects of different interventions, such as Indoor Residual Spraying (IRS) and Insecticide-treated nets (ITNs) on malaria. ${ }^{10,11}$ However, most of these studies used effect measures of these interventions without combining these with malaria intervention coverage information. Unlike simply presenting measures of effect such as odds ratios, combining intervention coverage data with effect measures may enable one to directly show the extent to which malaria prevalence would change when these interventions are scaled up. Using several waves of nationally representative survey data in Zambia, we contribute to filling this gap. Apart from providing evidence for policymakers that would better guide efforts to malaria elimination, our study presents a
Correspondence: Mukumbuta Nawa, The University of Zambia. School of Public Health Ridgeway Campus, P. 0. Box 50110, Lusaka, Zambia. Tel: +260977392064

E-mail: mktnawa@gmail.com

Key words: Indoor Residual Spraying (IRS), Insecticide Treated Nets (ITN), Standard Housing.

Acknowledgements: We want to thank Dr John Miller and the team at the National Malaria Elimination Centre of the Ministry of Health in Zambia for sharing the Malaria Indicator Survey datasets, which we used for this study. Prof Andrew P. Morse and Dr Cyril Caminade of the University of Liverpool who provided me with climatic data for Zambia. We also thank Prof Ingvild Sandoy of the University of Bergen.

Contributions: MN did the conceptualization, developed the proposal, obtained the datasets, did the analysis, made the initial draft manuscript. PH guided data analysis and contributed to writing the paper. $\mathrm{HH}$ reviewed the proposal and Manuscript. All authors approved the final manuscript.

Conflict of interest: The authors declare no conflict of interest.

Funding: None.

Ethical considerations and informed consent: ERES Converge Institutional Review Board approved this study (IRB) Ref No. 2017-Aug005 and by the National Health Research Authority (NHRA). The Ministry of Health $(\mathrm{MoH})$ also granted permission to access the datasets for this study. This study did not deal with individual respondents but anonymised datasets, so there was no need for individual respondents' consent. The data was anonymized by the NMEP who removed all personal identifiers and household GPS, so we only handled data with coded IDs without the code keys.

Received for publication: 19 April 2019.

Revision received: 23 August 2019.

Accepted for publication: 17 October 2019.

This work is licensed under a Creative Commons Attribution NonCommercial 4.0 License (CC BY-NC 4.0).

(C) Copyright: the Author(s),2020

Licensee PAGEPress, Italy

Journal of Public Health in Africa 2020; 11:1096 doi:10.4081/jphia.2020.1096

more informative method of assessing program impact that can be replicated in other contexts and countries. 


\section{Materials and Methods}

\section{Study design}

This study used data from repeated cross-sectional survey data from the Malaria Indicator Survey (MIS) for 2010, 2012 and 2015. The study focused on all children aged below five years (from zero to 59 Months) that were captured during the MIS 2010, 2012 and 2015. The MIS uses a two-stage cluster sampling method. The first stage sampling is based on clusters -25 631 Standard Enumeration Areas (SEAs) which partitions the whole country, and are based on the 2010 Census of Population and Housing. In 2010, 180 SEAs were sampled, 160 in 2012, and 150 in 2015. In the second stage, 25 households are sampled from each SEA, and all household members were included in the survey. ${ }^{3,12,13}$ The MISs are set at $95 \%$ confidence level, $80 \%$ power, the design effect of 2 and adjusted for a $20 \%$ non-response rate and are representative at national, provincial, urban and rural level.

\section{Study settings}

Zambia is one of the countries in the southern part of Sub-Saharan Africa (SSA). With a population of about 17 million, of which $40 \%$ is urban. It is one of the most urbanized countries in SSA. Although it is classified as a lower-middle-income country, there is high inequality, with more than $60 \%$ of the population living below the national poverty line. Access to health facilities is also hard, particularly among rural communities. Improvised housing structures, which allow free entry and exit of malaria vector mosquito, are more prevalent in rural areas than in urban areas. During the 2015 MIS survey, only $8 \%$ of households in rural areas had standard housing structures compared to $39 \%$ in urban areas while the overall was only at $14 \%{ }^{3}$ Standard housing is defined by solid roofs, such as concrete, iron or asbestos, brick walls, and concrete or tiled floors. Sub-standard structures were improvised houses such as grass-thatched roofs and mud or grass walls. ${ }^{9}$

The country has a tropical climate and a high burden of malaria, ${ }^{9}$ it is administratively divided into ten provinces. Malaria is highest in the northern parts, where prevalence was as high as $32 \%$ in children aged below five years. In the central regions, the disease is moderate, averaging $14 \%$, while it is lowest in the southern parts where prevalence was $0.6 \%$ as of $2015 .{ }^{3}$ In the east to west direction of the country, there are no significant differences in malaria prevalence. It is probably because rainfall patterns follow latitudes, which change in the north to the southern direction of the country. ${ }^{9}$ The burden of disease is also unequally concentrated on the poor. For example, fever among children less than five years is unfairly concentrated among the poor. There has not been any reduction in inequality between 2007 and 2014. ${ }^{14}$

Spending on Malaria has reduced recently; for example, the recent National Health Accounts show that nominal expenditure on malaria declined by almost $40 \%$ between 2015 and 2016, from $\$ 172$ million to $\$ 123$ million. However, Malaria accounts for the second-biggest share of health expenditure by disease area after HIV and AIDS. ${ }^{15}$

\section{Data}

For this study, datasets from the repository at the National Malaria Elimination Centre of the Ministry of Health in Stata 13 format for 2015 datasets and Microsoft Access 2010 for 2010 and 2012 datasets were obtained. The datasets were exported from the hosting database formats MS Access and Stata 13, respectively into MS Excel 2010. The column names and formats were then standardized in MS Excel 2010.
We then imported the datasets into Stata version 15, appended and saved as .csv files. ${ }^{16}$ The data was then cleaned by excluding all persons five years and older before we did the analysis.

\section{Data analysis}

We reported basic descriptive statistics such as mean, median, standard deviation and interquartile range for continuous variables. Categorical data were summarized using counts and percentages. We then fitted a multiple logistic regression model, which accounted for complex survey design in Stata version 15. We used a priori subject knowledge to establish the coefficients of predictor variables. This model was then used to obtain marginal predictions for malaria prevalence for 2015 . The predicted value for 2015 was compared against the observed values in MIS 2015 using a onesample test of proportions to assess the robustness of the model.

Further, a counterfactual analysis using hypothetical values in the model to predict how malaria prevalence would respond to varying levels of coverage of malaria inter-
2010 survey

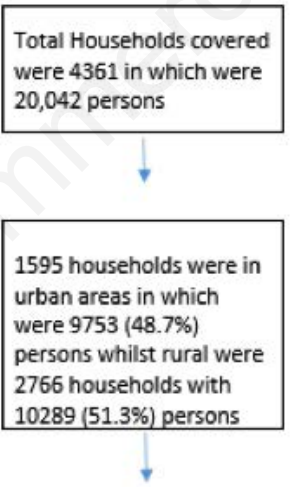

$7612(38.0 .0 \%)$ were male persons whilst 10 $289(62.0 \%)$ were female persons
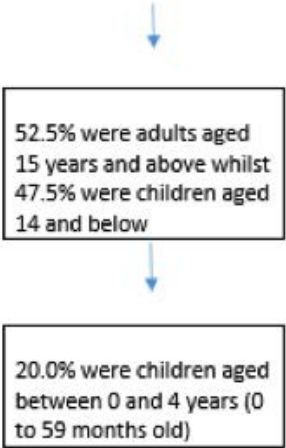

2012 survey

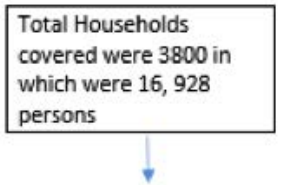

750 households were in urban areas in which were $3,425(20.2 \%)$ persons whilst rural were 3050 households with $13,503(79.8 \%)$ persons

\section{$8053(47.6 \%)$ were male persons whilst $8874(52.4 \%)$ were} female persons
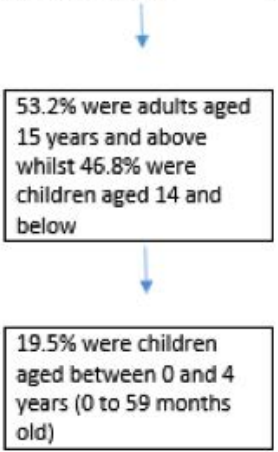

2015 survey
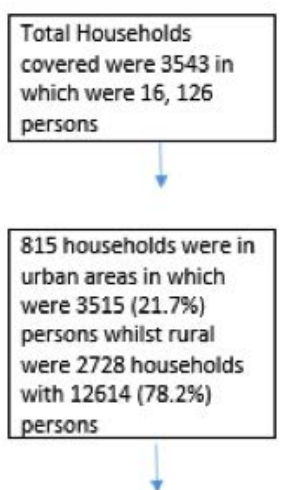

$7520(46.6 \%)$ were male persons whilst $8606(53.4 \%)$ were female persons

\begin{tabular}{l}
$\begin{array}{l}53.6 \% \text { were adults aged } \\
15 \text { years and above } \\
\text { whilst } 46.4 \% \text { were } \\
\text { children aged } 14 \text { and } \\
\text { below }\end{array}$ \\
$\qquad \begin{array}{l}17.5 \% \text { were children } \\
\text { aged between } 0 \text { and } 4 \\
\text { years (0 to } 59 \text { months } \\
\text { old) }\end{array}$ \\
\hline
\end{tabular}

Figure 1. Baseline characteristics of respondents. 
ventions was done. Finally, the country was stratified into three malaria epidemiological zones based on the WHO Global Technical Strategy (GTS). ${ }^{8}$ We then applied the counterfactual analysis in each of the epidemiological zones to address the effect modification in low, medium and high burden zones.

\section{Ethical considerations}

ERES Converge Institutional Review Board approved this study (IRB) Ref No. 2017-Aug-005 and by the National Health Research Authority (NHRA). The Ministry of Health $(\mathrm{MoH})$ also granted permission to access the datasets for this study. This study did not deal with individual respondents but anonymised datasets, so there was no need for individual respondents' consent. The data was anonymised by the NMEP who removed all personal identifiers and household GPS, so we only handled data with coded IDs without the code keys.

\section{Results}

\section{Basic demographic characteristics of respondents}

The response rates for all the three ${ }^{3}$ surveys were more than $95 \%$. The demographics were comparable across the studies. Figure 1 summarises the demographic characteristics of the participants.

\section{Model estimation of 2015 malaria prevalence observed by malaria indicator survey}

Table 1 summarises the coefficients, confidence intervals and the respective $\mathrm{P}$ values of the logistic regression model, which was the basis for the obtained marginal effects used in estimating malaria prevalence. Using these post-estimation margins, we were able to predict the 2015 Malaria Prevalence at national and provincial levels, which were very close to the observed values of malaria prevalence in 2015. The only exceptions were provinces with values of malaria prevalence of less than $10 \%$. Table 2 shows observed values in 2015 Malaria Indicator Survey, the predicted values using the post-estimation margins of the model and respective P-values for comparisons of the observed 2015 MIS prevalence and predicted values.

\section{Counterfactual analysis of malaria prevalence by varying levels of interventions}

Figure 2 shows how malaria prevalence varied with different levels of coverage of IRS, ITN and Standard housing while keeping all other variables constant at 2015 levels for the whole country. Malaria prevalence would reduce from $20.5 \%$ (95\% CI $10.4-30.6 \%$ ) when IRS coverage is at $10 \%$ to $16.0 \%(6.8-25.3 \%)$ when IRS is at $90 \%$. When ITN utilisation the previous night among children aged below five years is varied from $10 \%$ to $90 \%$, malaria prevalence may reduce from $20.8 \%$ (95\%CI 10.1 $-31.6 \%)$ to $18.4 \%$ (95\% CI $9.1-27.7 \%)$ respectively. Further, malaria prevalence would reduce from $19.6 \%$ (95\% CI 9.8 $29.7 \%$ ) when standard housing is at $10 \%$ to 12.4 (95\% CI 4.6 - 20.2) when standard house is at $90 \%$. Finally, we also presented the best-case scenario where IRS and ITNs were fixed at $80 \%$ coverage, and standard housing was varied while keeping all other variables constant at 2015 levels. Prevalence reduced from $16.3 \%$ (95\%CI $7.2-25.4 \%)$ when standard accommodation was at $10 \%$, to $10.1 \%(96 \%$ CI $3.3-$ $16.9 \%$ ) when standard accomodation was at $90 \%$. With the latter scenario, prevalence

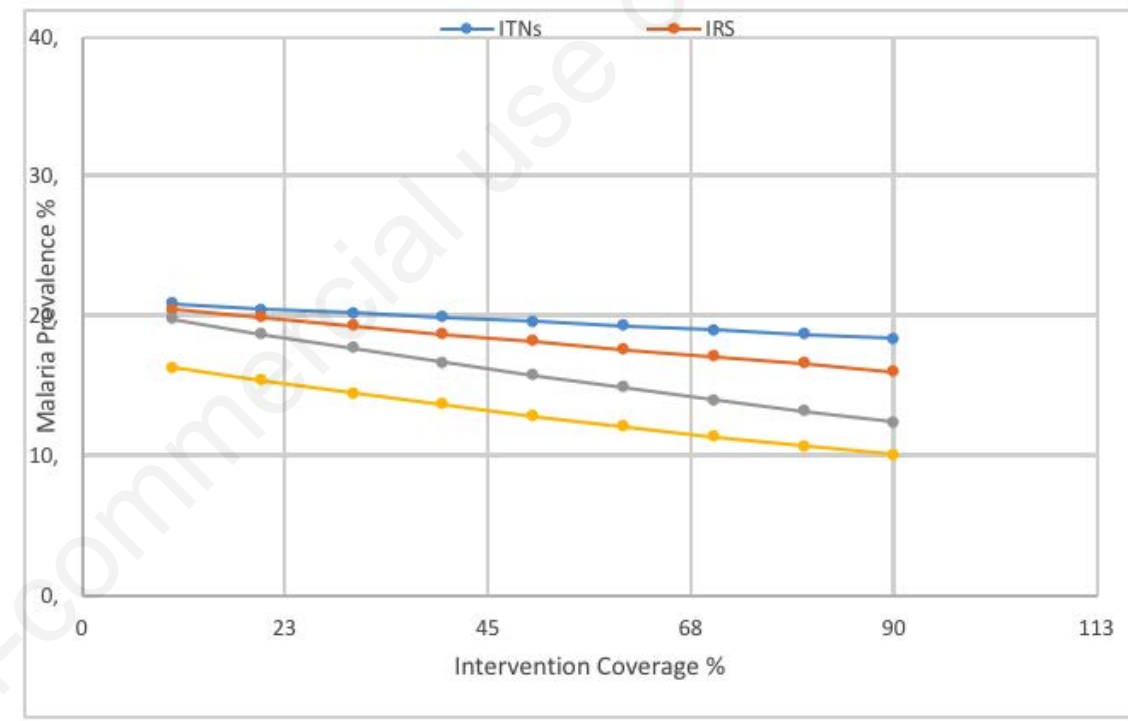

Figure 2. Varying ITNs, IRS and Standard house while keeping all other variables constant at $\mathbf{2 0 1 5}$ for the whole country.

Table 1. Coefficients from a logistic regression model.

\begin{tabular}{|c|c|c|c|c|}
\hline Variable $\left(\mathrm{X}_{\mathrm{i}}\right)$ & Coefficient & \multicolumn{2}{|c|}{$95 \%$ Conf. Interval } & P-Value \\
\hline Age category (12-59 months) & 1.563 & 1.184 & 1.942 & $<0.001$ \\
\hline Sex (females) & -0.100 & -0.278 & 0.078 & 0.270 \\
\hline Residence location (urban) & -1.496 & -1.950 & -1.042 & $<0.001$ \\
\hline Altitude (meters) & -0.001 & -0.001 & 0.000 & 0.065 \\
\hline Standard house (yes) & -0.693 & -1.031 & -0.354 & $<0.001$ \\
\hline Wealth status (not poorest quartile) & -0.411 & -0.640 & -0.182 & $<0.001$ \\
\hline Indoor residual sprayed (yes) & -0.376 & -0.673 & -0.079 & 0.013 \\
\hline Slept under a net (yes) & -0.196 & -0.352 & -0.039 & 0.015 \\
\hline Rainfall (mm) & 0.001 & -0.001 & 0.003 & 0.302 \\
\hline Temperature $\left({ }^{\circ} \mathrm{C}\right)$ & -0.323 & -0.456 & -0.190 & $<0.001$ \\
\hline cons & 5.239 & 1.242 & 9.236 & 0.010 \\
\hline
\end{tabular}


reduced to the moderate level of less than $15 \%$ when standard accommodation was at $25 \%$ where it is permissible to commence elimination activities. Finally, the worstcase scenario was assumed where interventions like ITNs and IRS were reduced to $10 \%$. All other variables remained constant at 2015 levels, and prevalence would increase from 2015 's 19.4 to 22.0 (95\% CI $11.0-33.1 \%)$.

The situation presented in Figure 2 used the dataset for the whole country. However, Zambia shows heterogeneity in prevalence, with some provinces showing low prevalence below five per cent in children below the age of five years, namely Southern and Lusaka provinces. Four other areas, namely Eastern, Central, Copper-belt and Western provinces, showed a moderate prevalence of between five and fifteen per cent. Four more regions, namely Luapula, Muchinga, Northern and North-western, had a higher prevalence of more than $15 \%$ in children aged below five years. The framework used to categorise the provinces into low, moderate and high endemic areas is the WHO Global Technical Strategy for malaria elimination (GTS). ${ }^{8}$ We then did simulations of the effects of three interventions in these three malaria epidemiological zones.

\section{Effects in the low malaria epidemio- logical zone}

The estimated mean prevalence for Lusaka and Southern provinces was 1.3\% (95\% CI, $0.6 \%-3.1 \%)$ while the individual provincial prevalence in 2015 was $2.4 \%$ and $0.6 \%$ respectively. The marginal effect of ITNs in this zone was $-0.008(95 \% \mathrm{CI}$, $0.0198,0.0038$; P-value $=0.180)$, whilst that of IRS was - $0.052(95 \% \mathrm{CI},-0.037$, 0.007 ; P-value $=0.178)$ and that of standard housing was -0.010 (95\% CI, -0.030, 0.009; $\mathrm{P}$-value $=0.300)$. All three interventions did not have statistically significant effects on malaria prevalence in this epidemiological zone. Figure 3 shows the effects of varying ITN, IRS and standard housing in the low malaria epidemiological zone.

\section{Effects in the moderate malaria epi- demiological zone}

The estimated mean prevalence in the moderate malaria epidemiological zone was $14.1 \%$ (95\% CI, $10.3 \%-18.0 \%)$. The marginal effect of ITNs in this zone was $0.019(95 \%$ CI, $-0.054,0.016$; P-value $=$ $0.301)$, whilst that of IRS was - 0.030 (95\%CI, -0.060, 0.009; P-value $=0.133$ ) and that of standard housing was -0.029 (95\%CI, -0.078, 0.020; P-value $=0.248$ ). All three interventions did not have statistically significant effects on malaria prevalence in this epidemiological zone. Figure 4 shows the effects of varying ITN, IRS and standard housing in the moderate malaria epidemiological zone.

\section{Effects in the high malaria epidemi- ological zone}

The estimated mean prevalence in the high malaria epidemiological zone was

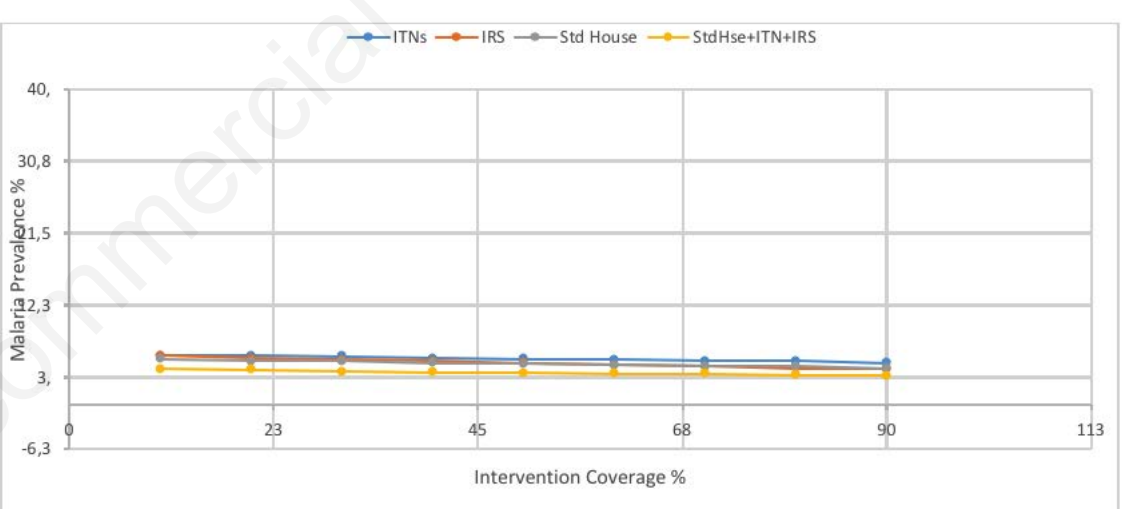

Figure 3. Varying ITNs, IRS and Standard house while keeping all other variables constant at 2015 for Low Prevalence Provinces.
$28.7 \%(95 \%$ CI, $23.3 \%-34.1 \%)$. The marginal effect of ITNs in this zone was $0.041(95 \%$ CI, $-0.074,-0.009 ;$ P-value $=$ $0.012)$, whilst that of IRS was - 0.094 $(95 \%$ CI $,-0.179,-0.008 ;$ P-value $=0.031)$ and that of standard housing was -0.156 (95\%CI, -0.246, -0.066; P-value $=0.001)$. nificant effects on malaria prevalence in the high malaria epidemiological zone. Figure 5 shows the effects of varying ITN, IRS and standard housing in this epidemiological zone.

\section{Discussion}

This study demonstrates that using already available MIS data; we can model targeted coverage levels for control interventions to reach the elimination threshold. This tool is useful for planning of national malaria control. Key findings indicate that the effects of interventions like ITN, IRS and standard housing are more pronounced in the high malaria epidemiological zones. That there is a need for increased coverage All three interventions had statistically sig-

Table 2. Observed 2015 prevalence $v$ s predicted 2015 malaria prevalence.

\begin{tabular}{llcccc} 
& Province & Observed & Predicted (95\%Cl) & P-value & No. of Children \\
1 & Central & 13.8 & $15.9(7.5-24.2)$ & 0.386 & 203 \\
2 & Copper-belt & 15.2 & $17.8(8.6-27.1)$ & 0.198 & 316 \\
\hline 3 & Eastern & 12.7 & $13.6(5.1-22.1)$ & 0.683 & 229 \\
4 & Luapula & 32.5 & $30.2(16.9-43.5)$ & 0.244 & 562 \\
\hline 5 & Lusaka & 2.4 & $8.9(3.9-14.0)$ & $<0.000$ & 198 \\
6 & Muchinga & 31.4 & $24.1(11.5-36.7)$ & 0.034 & 181 \\
\hline 7 & Northern & 27.6 & $27.3(14.3-40.3)$ & 0.909 & 293 \\
8 & North-Western & 22.6 & $27.0(14.2-39.9)$ & 0.113 & 227 \\
\hline 9 & Southern & 0.6 & $15.0(6.1-23.8)$ & $<0.000$ & 281 \\
10 & Western & 15.6 & $17.9(7.8-28.0)$ & 0.329 & 237 \\
\hline
\end{tabular}


of primary interventions such as ITN and IRS to at least $90 \%$. Introduction of improving housing structures to standard housing would augment the fight against malaria.

Studies have shown that global malaria is declining, mainly due to interventions, even though not all the decline can be explained. ${ }^{17}$ Models have been developed for forecasting malaria, some of which are based on climatic conditions like temperature, rainfall and vegetative indices. ${ }^{18,19}$ These models are resource-intensive and require a lot of technical equipment and expertise. They are also helpful in mapping malaria transmission. The model in this publication offers a less resource-intensive approach focusing on average estimates generating targets for malaria control that can guide programmatic activities. The model also controls for the effect of rainfall and temperature, but currently does not include humidity. ${ }^{20}$

Other models use historical data from routine surveillance sources and apply some versions of time series, such as Autoregressive Integrated Moving Averages (ARIMA). Such models have been shown to help in forecasting malaria burden, including seasonal variations even in high burden areas. Programs can then prepare for ongoing malaria transmission and adequately prepare inputs to avoid stock-outs of drugs, test kits, and other supplies. ${ }^{21,22}$ The model used in this study combines age, sex, environmental and sociodemographic variables and also includes a constant, which encompasses the unaccounted for residuals that have been generated using data from the recent three Malaria Indicator Surveys in Zambia. This model can be useful in strategic planning for target setting where multiple interventions are used in malaria programs. For example, if a malaria program wants to reduce prevalence by $50 \%$, it can estimate the coverage in predictor variables that would result in the desired $50 \%$ reduction in prevalence by changing coverage levels in the model.

To remain realistic, only variables that can feasibly be changed using program implementation such as IRS, ITNs and Housing were varied while keeping variables that cannot be feasibly changed in a malaria program constant at 2015 levels. For example, urban/rural residence location: one cannot move people from rural areas to urban areas just because urban areas have less risk of malaria compared to rural areas. Similarly, one cannot change temperature, altitude or people's wealth status in a malaria intervention program. A malaria program can, however, spray people's houses, distribute nets and promote policies for the building of standard houses and installation of fly screens in dwellings. The advantage of this tool is that it uses existing data such as Malaria Indicator Surveys (MIS) and weather conditions data from the satellite-generated climate database called Climate Explorer (https://clime.xp.knmi.nl), which are not domiciled in Zambia, to predict malaria reductions at provincial and national levels. Its shortcoming is that it relies on household surveys data such as MISs, which are only done periodically and therefore cannot be used for short term monitoring of progress. It can be used for strategic planning to plan certain levels of coverage of interventions that would bring down malaria. Then as a complementary tool some time series like Autoregressive Integrated Moving Averages (ARIMA) models that use routine data from them for monitoring progress in between surveys. Zambia can benefit from this modelling approach, especially in high endemic, where most of the malaria cases occur.

Out of the five hypothetical scenarios that were created in the counterfactual analysis where ITNs, IRS and standard housing were varied using the dataset for the whole country; no situation was able to predict the desired prevalence of less than $5 \%$. Even when ITNs and IRS were fixed at $80 \%$ and standard housing was varied up to $90 \%$, which predicted the lowest prevalence of $10 \%$. Based on the WHO Global Technical Strategy (GTS) for malaria elimination, level four (prevalence $>15 \%$ ) where most of Zambia was in 2015. Elimination activities may not be implemented as there was still a need to bring down the prevalence first using vector control and facility case management. When prevalence gets to level

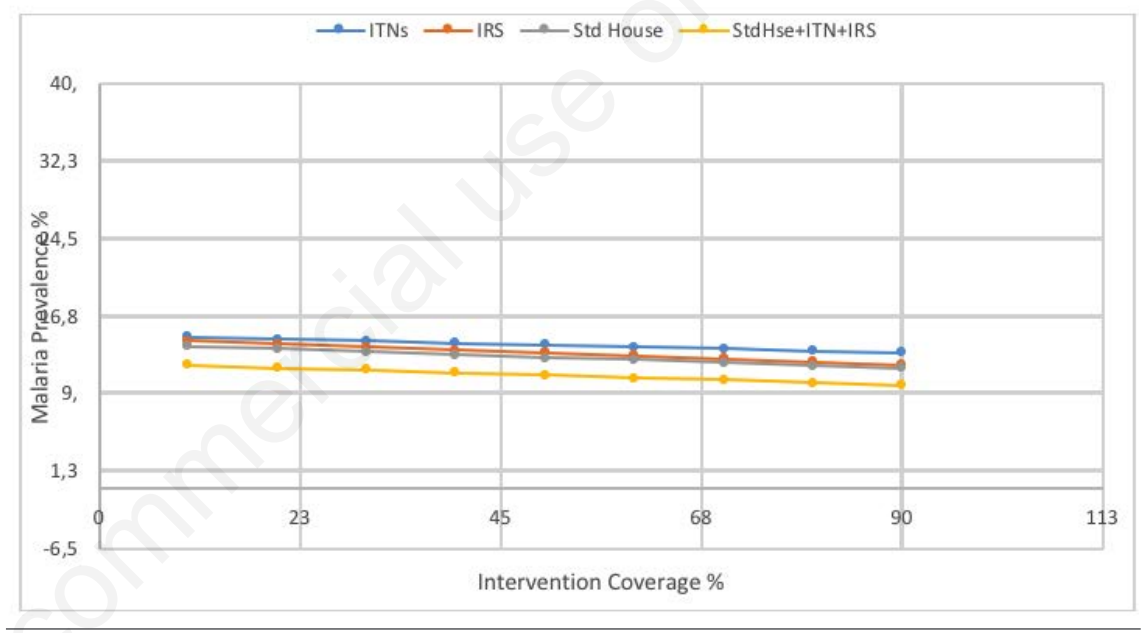

Figure 4. Varying ITNs, IRS and Standard house while keeping all other variables constant at 2015 for Medium Prevalence Provinces.

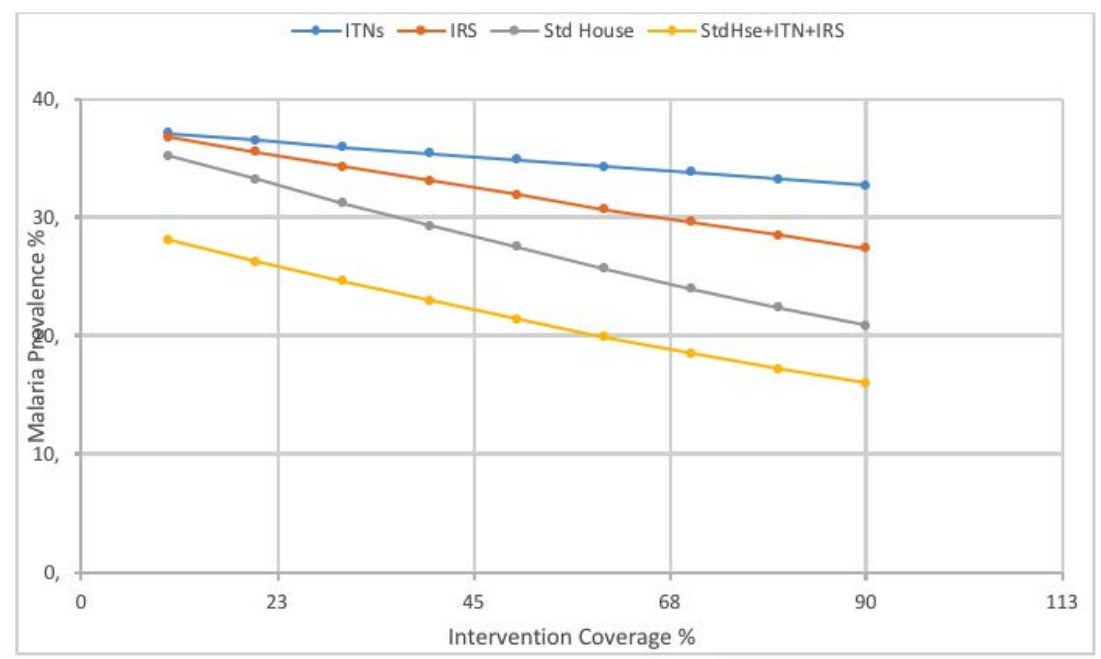

Figure 5. Varying ITNs, IRS and Standard house while keeping all other variables constant at $\mathbf{2 0 1 5}$ for High Prevalence Provinces. 
three, where it lies between $15 \%$ and $5 \%$, accelerator activities such as Mass Drug Administration (MDA) and community case management targeted at hotspots of transmission may be implemented in areas that have the capacity. Otherwise, these are recommended when prevalence is at levels two or below, where prevalence is lower than $5 \%$. In Zambia, only two provinces had prevalence below 5\% namely Lusaka and Southern provinces as of 2015. Accelerator interventions such as MDA and reactive case detection have been implemented in these areas to drive the elimination agenda at the subnational level. ${ }^{23}$ The primary challenge to subnational elimination efforts is contamination through the importation of malaria cases within regions. There are no restrictions or malaria screening in the mobility of persons across provinces within the country. For example, one study found that $94 \%$ of malaria cases at health facilities in Lusaka district were imported from other provinces. The frequency of travel and duration of stay in malaria areas were found to be significant risk factors among residents. ${ }^{24}$ Due to the high risk of continual contamination because of human mobility within the country, the authors perceive that a subnational approach to elimination is not sustainable. Instead, bringing down prevalence in all provinces and implementing the accelerator interventions to drive down local transmission further while screening people entering at borders and airports may be a more feasible approach.

This study was able to predict a coverage that would bring prevalence to at least level three using the dataset for the whole country. In one scenario; ITNs and IRS were kept constant at 2015 levels, standard housing had to reach at least $60 \%$, which in the view of the authors may not be very feasible, given that Zambia is a resource-limited country. In 2015, standard housing was at only $13 \%$ of the surveyed households. On the other hand, when mainstream interventions such as ITNs and IRS are increased to $80 \%$ from 2015 levels of $49 \%$ and $29 \%$ respectively, level three was reached when standard housing reached only $20 \%$ which is more feasible from the $13 \%$ of recorded in 2015. Keeping other variables constant and varying either ITNs or IRS alone did bring prevalence below $15 \%$ neither did increase both ITN and IRS at the same time to $80 \%$.

To address effect modification; the country was divided into three epidemiological zones based on the WHO GTS framework. While ITNs, IRS and standard housing were shown to bring down malaria prevalence as coverage was increased, the effects were not statistically significant in low and medium malaria epidemiological zones, but were significant in the high malaria epidemiological zone. To the best of the knowledge of the authors, this is the first time this finding has been demonstrated in Zambia. However, it is in agreement with one study done in Uganda, which also found that the effects of interventions varied within regions. ${ }^{25}$ While Zambia had carried out nationwide ITN mass distribution campaigns in $2014^{26}$ and 2017 , this study brings to light at least a need to review this approach. One study recommended a prioritization approach to the allocation of resources at the subnational level to achieve efficiencies. ${ }^{27}$

The results from the present study seem to suggest that in all epidemiological zones, standard housing had more effects on bringing down malaria prevalence followed by IRS and least was ITNs. It may not be feasible to construct standard houses for all people in a resource-constrained country like Zambia. However, it could be possible to modify housing structures towards insect-proofing. It may not also be efficient to spray all housing structures with residual insecticides as it has been shown to work better on painted walls compared to rough walls. ${ }^{28}$ ITNs, on the other hand, have been shown to have little or no effect on malaria prevalence, particularly in low malaria epidemiological settings. ${ }^{29}$ But other interventions such as MDA and community case management are effective in low endemicity settings. ${ }^{30,31}$ ITNs work by either killing or repelling invading vectors. If the ITNs are repelling than killing, it has been demonstrated elsewhere that this may pose an increased risk to non-users ${ }^{32}$ or change in behaviour to biting at dusk and dawn. ${ }^{33}$

\section{Conclusions}

The present study has shown that malaria prevalence can be predicted using different levels of coverage of control interventions while controlling for Age, Sex, rainfall, temperature and Socioeconomic variables such as type of housing and wealth index, and the results were comparable to the actual field data from the National Malaria Indicator Survey of 2015. This modelling approach can help the country as it predicts within acceptable precision in the moderate and high endemic areas. As of 2015 , only two out of the ten provinces were in the low endemic zones, where the model was not accurate.

Among critical interventions, IRS, ITN and standard housing at appropriate coverage levels have been shown to have poten- tial to reduce malaria prevalence from current high levels in most parts of the country to at least moderate levels where accelerator interventions for elimination may be implemented. The effects of reducing malaria prevalence were noted to be more pronounced in high prevalence zones of the country compared to low burden areas implying that there might be a need for intervention prioritisation at least at provincial level based on what works locally.

\section{Recommendations}

There is a need to increase coverage of mainstay interventions such as ITNs and IRS from 2015 levels, especially in the high prevalence epidemiological zones.

Current mainstay interventions such as ITN and IRS alone are effective but have not been shown to bring down malaria to at least moderate levels. There is a need to complement them with housing infrastructure improvements from improvised housing structures that allow free entry of vector mosquito to standard housing structures that are mosquito proof. In line with the WHO GTS recommendations, it can be possible then to implement accelerator interventions such as enhanced vector control, Mass Drug administration, community case management and surveillance once prevalence is brought down to at least moderate levels.

The modelling approach used in this study can be used by malaria programs to inform target setting and intervention selection for locally effective programs.

\section{Limitations}

Due to inadequate numbers of respondents who received treatment in the two weeks preceding the surveys, prompt treatment on malaria prevalence could not be included in the model.

The model used in this study was able to predict malaria prevalence accurately within 95\% confidence intervals for moderate and high endemic areas. It was not accurate for low endemic areas with prevalence below $5 \%$ in children aged below five years.

\section{References}

1. Masaninga F, Chanda E, ChandaKapata P, et al. Review of the malaria epidemiology and trends in Zambia. Asian Pac J of Trop Biomed 2013;3:8994.

2. Fink G, Masiye F. Health and agricultural productivity: Evidence from Zambia. J Health Econ 2015;42:151-64.

3. Ministry of Health M, Central Statistics Office, (CSO), PATH Malaria Control 
and Evaluation Partnership in Africa (MACEPA), the United States President's Malaria Initiative (PMI), the World Bank, UNICEF, and the World Health Organization, (WHO). Malaria Indicator Survey. Lusaka: Ministry of Health; 2015.

4. Schumacher R-F, Spinelli E. Malaria in children. Mediterr J Hematol Infect Dis 2012;4:e2012073-e.

5. Akiyama T, Pongvongsa T, Phrommala $\mathrm{S}$, et al. Asymptomatic malaria, growth status, and anaemia among children in Lao People's Democratic Republic: a cross-sectional study. Malar J 2016;15:499.

6. Chirwa TG, Odhiambo NM. Sources of Economic Growth in Zambia: An Empirical Investigation. Global Bus Rev 2017;18:275-90.

7. Republic of Zambia, Ministry of Health. National Malaria Elimination Strategic Plan 2017 - 2021. 2017.

8. RBM. RBM Partnership Strategic Plan 2018 - 2020. Geneva: 2018.

9. Nawa M, Hangoma P, Morse AP, Michelo C. Investigating the upsurge of malaria prevalence in Zambia between 2010 and 2015: a decomposition of determinants. Malar J 2019;18:61.

10. Sharp B, Van Wyk P, Sikasote JB, et al. Malaria control by residual insecticide spraying in Chingola and Chililabombwe, Copperbelt Province, Zambia. Trop Med Int Helath 2002;7:732-6.

11. Choi HW, Breman JG, Teutsch SM, et al. The effectiveness of insecticideimpregnated bed nets in reducing cases of malaria infection: a meta-analysis of published results. Am J Trop Med Hyg 1995;52:377-82.

12. Ministry of Health CSO, PATH, Malaria Control and Evaluation Partnership in Africa (MACEPA) tUS, President's Malaria Initiative TWB, UNICEF, and the World Health Organization. Malaria Indicator Survey 2012. 2012.

13. Ministry of Health CSO, PATH, Malaria Control and Evaluation Partnership in Africa (MACEPA) tUS,
President's Malaria Initiative TWB, UNICEF, and the World Health Organization. Malaria Indicator Survey 2010. 2010.

14. Hangoma P, Aakvik A, Robberstad B. Explaining changes in child health inequality in the run-up to the 2015 Millennium Development Goals (MDGs): The case of Zambia. PLOS ONE. 2017;12:e0170995.

15. Knoema. National Health Accounts 2019. Available from https://knoema.com/ WHONHA2019 /national-health-accounts? country=1000470-zambia.

16. StataCorp. Stata Version 152018. Available from https://www.stata.com/

17. Nkumama IN, O'Meara WP, Osier FHA. Changes in Malaria Epidemiology in Africa and New Challenges for Elimination. Trends Parasitol 2017;33:128-40.

18. Alegana VA, Atkinson PM, Wright JA, et al. Estimation of malaria incidence in northern Namibia in 2009 using Bayesian conditional-autoregressive spatial-temporal models. Spat Spatiotemporal Epidemiol 2013;7:2536.

19. Haque U, Magalhães RJS, Reid HL, et al. Spatial prediction of malaria prevalence in an endemic area of Bangladesh. Malar J 2010;9:120.

20. MacLeod DA, Jones A, Giuseppe FD, et al. Demonstration of successful malaria forecasts for Botswana using an operational seasonal climate model. Environ Res Lett 2015;10:044005.

21. Anokye R, Acheampong E, Owusu I, Isaac OE. Time series analysis of malaria in Kumasi: Using ARIMA models to forecast future incidence. Cogent Soc Sci 2018;4:1461544.

22. Briët OJT, Amerasinghe PH, Vounatsou P. Generalized Seasonal Autoregressive Integrated Moving Average Models for Count Data with Application to Malaria Time Series with Low Case Numbers. Plos One. 2013;8:e65761.

23. Larsen DA, Chisha Z, Winters B, et al. Malaria surveillance in low-transmission areas of Zambia using reactive case detection. Malar J 2015;14:465.

24. Lowa M, Sitali L, Siame M, Musonda P. Human mobility and factors associated with malaria importation in Lusaka district, Zambia: a descriptive cross-sectional study. Malar J 2018;17:404.

25. Ssempiira J, Nambuusi B, Kissa J, et al. The contribution of malaria control interventions on Spatio-temporal changes of parasitaemia risk in Uganda during 2009-2014. Parasite Vector 2017;10:450.

26. Masaninga F, Nawa M, Ndhlovu K, et al. Insecticide-treated nets mass distribution campaign: benefits and lessons in Zambia. Malar J 2018;17:173.

27. Walker PGT, Griffin JT, Ferguson NM, Ghani AC. Estimating the most efficient allocation of interventions to achieve reductions in Plasmodium falciparum malaria burden and transmission in Africa: a modelling study. Lancet Glob Health. 2016;4:e474-e84.

28. Desalegn Z, Wegayehu T, Massebo F. Wall-type and indoor residual spraying application quality affect the residual efficacy of indoor residual spray against wild malaria vector in southwest Ethiopia. Malar J 2018;17:300.

29. Steinhardt LC, Jean YS, Impoinvil D, et al. Effectiveness of insecticide-treated bednets in malaria prevention in Haiti: a case-control study. Lancet Glob Health. 2017;5:e96-e103.

30. Poirot E, Skarbinski J, Sinclair D, et al. Mass drug administration for malaria. Cochrane Database Syst Rev 2013;12: Cd008846.

31. Johnson AD, Thiero O, Whidden C, et al. Proactive community case management and child survival in periurban Mali. BMJ Global Health 2018;3: e000634.

32. Gu W, Novak RJ. Predicting the impact of insecticide-treated bed nets on malaria transmission: the devil is in the detail. Malar J 2009;8:256.

33. Thomsen EK, Koimbu G, Pulford J, et al. Mosquito Behavior Change After Distribution of Bednets Results in Decreased Protection Against Malaria Exposure. J infect dis 2017;215:790-7. 